\title{
Efficient Interface Conditions for the Finite Difference Beam Propagation Method
}

\author{
H. J. W. M. Hoekstra, G. J. M. Krijnen, and P. V. Lambeck
}

\begin{abstract}
It is shown that, by adapting the refractive indexes in the vicinity of interfaces, the 2-D beam propagation method based on the finite difference (FDBPM) scheme can be made much more effective. This holds especially for TM modes propagating in structures with high-index contrasts, such as surface polaritons. A short discussion is given on the applicability of the FDBPM.
\end{abstract}

\section{INTRODUCTION}

B EAM propagation methods (BPM) are widely used in integrated optics to simulate the behavior of light in more or less complicated wave guiding structures. Many papers on the BPM based on the fast Fourier transform (FFTBPM) have been published the last ten years (see [1]-[3], and references therein). Recently a propagation method based on a finite difference scheme (FDPBM), assuming the so-called slowly varying envelope approximation (SVEA), has been introduced [4]-[6]. The FFTBPM is reliable for structures having not too high-index contrasts, and, in the presence of such contrasts, the propagation should be paraxial [1], [2]. For the FDBPM the only requirement is that the differences between the effective indexes of the propagating modes are small. The latter is due to the SVEA and will be discussed below. So, the FDBPM can be used in the presence of high index contrasts, but paraxial propagation is still a, possibly less stringent (if compared to FFTBPM), requirement.

As large index contrasts are allowed in the FDBPM scheme also TM modes come into play. We have found that, if no special arrangements are made, the lateral step size, $\Delta x$, should be extremely small for TM modes propagating in structures with high index contrasts such as occurring in the presence of a metal layer. Further, errors may be introduced by the fact that in the BPM, generally using a discretization of the computational window, things cannot always be arranged such that the true interfaces coincide with a mesh point. Below we will show that the accuracy of the effective mode index, and therefore the distribution of the propagating field, can be improved considerably (for a given value of $\Delta x$ ) by adapting the refractive index at mesh points in the vicinity of interfaces. A necessary condition for this is that (for TM modes) the effective indexes of the modes involved are known, at least approximately. This may seem awkward for a BPM, but often the effective indexes can be estimated reasonably accurate; furthermore approximate knowledge of these indexes is a

Manuscript received October 23, 1991; revised March 31, 1992.

The authors are with MESA Research Institute, University of Twente, 7500 AE Enschede, The Netherlands.

IEEE Log Number 9202093 prerequisite for the use of the FDBPM algorithm. We remark that the introduction of the adapted indexes allows for larger values of $\Delta x$, and does not influence the result for the limit $\Delta x \rightarrow 0$. The method is illustrated by a few examples.

\section{THEORY}

\section{A. Calculational Scheme of the FDBPM}

Assuming an $\exp (i \omega t)$ time dependence, propagation mainly in the positive $z$-direction in a structure with a mean refractive index $n_{0}$, and using the SVEA it follows [4]:

$$
2 i k_{0} n_{0} \frac{\partial \psi}{\partial z}=\left\{\frac{\partial^{2}}{\partial x^{2}}+k_{0}^{2}\left[n^{2}(x, z)-n_{0}^{2}\right]\right\} \psi .
$$

Here it is assumed that the field $E_{y}=\psi \cdot \exp \left(-i k_{0} n_{0} z\right), \psi$ being a slowly varying function of $z$. In the case of TM modes, i.e., $H_{y}=\psi$. $\exp \left(-i k_{0} n_{0} z\right)$, the rhs of (1) should include the expression [7]

$$
-\frac{\partial \ln \left(n^{2}\right)}{\partial x} \cdot \frac{\partial \psi}{\partial x}
$$

Introducing a uniform discretization with step sizes $\Delta x$ and $\Delta z$, and integrating in the interval $[s \Delta z,(s+1) \Delta z]$, using averaged (mean of initial and final fields) values for the rhs (Crank-Nicolson) it follows [6]:

$$
\begin{gathered}
b_{j,-} \psi_{j-1}^{s+1}+a_{j,+} \psi_{j}^{s+1}+\psi_{j+1}^{s+1} \\
=-b_{j,-} \psi_{j-1}^{s}-a_{j,-} \psi_{j}^{s}-\psi_{j+1}^{s} .
\end{gathered}
$$

Here $a_{j,+/-}=-b_{j,+}+k_{0}^{2}\left(n_{j}^{2}-n_{0}^{2}\right)(\Delta x)^{2}-/+4 i k_{0} n_{0}(\Delta x)^{2} / \Delta z$, with $b_{j,-}=1+d_{j}, b_{j,+}=2+d_{j}$ and $d_{j}=0$ (TE) or $d_{j} \simeq\left(n_{j+1}^{2}-n_{j}^{2}\right) / n_{j}^{2}$ (TM; see also Section II-C). The suband superscripts used in (3) denote the $x$ - and the $z$-coordinate respectively, e.g., $\psi_{j}^{s}, j=0,1, \ldots, N$, is the slowly varying part of the field at $x=j . \Delta x$ and $z=s . \Delta z$. Equation (3) can be brought into the form:

$$
\mathbf{A} \vec{\psi}^{s+1}=\mathbf{f}, \operatorname{with}\left(\vec{\psi}^{s+1}\right)^{t} \equiv\left(\psi_{1}^{s+1}, \psi_{2}^{s+1} \ldots, \psi_{N-1}^{s+1}\right) .
$$

In (4) $\boldsymbol{A}$ is a tridiagonal matrix of order $N-1$, having upper subdiagonal elements equal to unity and lower subdiagonal elements, $b_{2,-}, b_{3,-}, \cdots, b_{N-1,-}$, which are also equal to unity except for TM polarization those elements corresponding to interfaces. The vector elements $\psi_{j}^{s+1}$ can be solved quite effectively in a standard fashion (see e.g., [8] p. 166) if (expressions for) the fields at the boundaries, $\psi_{0}^{s+1}$ and $\psi_{N}^{s+1}$ are known [6], [9]. 
In order to discuss the conservation of energy flow we assume real values for $n^{2}$ and $n_{0}^{2}$ and note that, neglecting the fields at the boundaries, (4) can be written in the following form [10]:

$$
(i c \mathbf{I}+\mathbf{B} \Delta z) \vec{\psi}^{s+1}=(i c \mathbf{I}-\mathbf{B} \Delta z) \vec{\psi}^{s}
$$

with $c \equiv 4 k_{0} n_{0}(\Delta x)^{2}$ and $I$ is the unit matrix. For TE polarization the matrix $B$ is real and symmetric and the eigenvectors of $\boldsymbol{B}$ are orthogonal. Then, using (5) it may be shown that the total energy flow per unit length, which is proportional to $\sum\left|\psi_{j}\right|^{2}$, is a constant of motion. For TM polarization it can be shown that the constant of motion is given by:

$$
\begin{gathered}
\sum\left|\psi_{j} / g_{i}\right|^{2} \text {, with } g_{1}=1 \\
\text { and } g_{j}^{2}=\Pi_{k=1}^{j-1} b_{k+1,-} \text { for } j=2, \cdots, N-1 .
\end{gathered}
$$

The summation in (6) is, in good approximation, proportional to $\sum\left|\psi_{j} / n_{j}\right|^{2}$, as it should according to standard waveguide theory. The transformation $\psi_{j} \rightarrow \psi_{j} / g_{j}$ corresponds to a similarity transformation of $B$ into a real, symmetric matrix [11]: $\boldsymbol{G}^{-1} \boldsymbol{B} \boldsymbol{G}$, where $\boldsymbol{G}$ is a diagonal matrix with $G_{j j} \equiv g_{j}$. So, in order to prevent unphysical energy loss or gain one should, for TM polarization, after each step in a $z$-variant part of the structure, perform the following correction:

$$
\psi_{j}^{s+1}=g_{j}^{s+1} \psi_{j, 0}^{s+1} / g_{j}^{s} .
$$

Here $\psi_{j, 0}^{s+1}$ has been calculated according to (4) and $g_{j}^{s}$ corresponds to the structure in the interval $[s \Delta z,(s+1) \Delta z]$.

\section{B. Applicability of the FDBPM}

We will discuss first the effect of the SVEA and the discretization along the $z$ axis (for TE polarization, the TM case being similar). It is assumed that the stepsize along the propagation direction, $\Delta z$, is small enough to approximate the structure, which may be irregular, sufficiently accurate For each such step the structure can be considered to be $z$-independent and the field pattern can be decomposed into modal fields. Consider a modal field, $\xi(x)$, corresponding to

$$
\frac{\partial^{2} \xi}{\partial x^{2}}+k_{0}^{2}\left(n^{2}-n_{e}^{2}\right) \xi=0
$$

$n_{e}$ being the effective index, which propagates according to the FDBPM algorithm. We suppose, for the moment, that this modal field is propagated correctly except with respect to the phase, i.e.,

$$
\begin{aligned}
E(x, z) & =\xi(x) \cdot \exp \left\{-i k_{0}\left(n_{e}+\Delta n\right) z\right\} \\
& =\psi(x, z) \cdot \exp \left\{-i k_{0} n_{0} z\right\} .
\end{aligned}
$$

Using (1), (8), (9) it follows:

$$
\Delta n=\left(n_{e}-n_{0}\right)^{2} /\left(2 n_{0}\right) .
$$

Substituting (9), using (10), into (1) it follows that $\psi$, defined by $(9)$ is indeed a solution of (1).

Equation (10) gives the error in the modal effective index due to the difference between the mode index and the background index, $n_{0}$. However, the nonvanishing step size, $\Delta z$, also introduces some error. Substituting (8)-(10) into (1) it follows:

$$
\begin{aligned}
\frac{\partial \psi}{\partial z} & =-i k_{0}\left\{\left(n_{e}-n_{0}\right)+\frac{\left(n_{e}-n_{0}\right)^{2}}{2 n_{0}}\right\} \psi \\
& =-i k_{0} \frac{\left(n_{e}^{2}-n_{0}^{2}\right)}{2 n_{0}} \psi \equiv-i \alpha \psi .
\end{aligned}
$$

Whence, integrating as discussed above (3)

$$
\psi^{s+1}-\psi^{s}=-i \alpha\left(\psi^{s+1}+\psi^{s}\right) \Delta z / 2
$$

so

$$
\psi^{s+1}=\left\{\frac{2-i \alpha \Delta z}{2+i \alpha \Delta z}\right\} \psi^{s} \equiv \psi^{s} \exp (-i \gamma \Delta z)
$$

whereas the solution according to (11) would be

$$
\psi^{s+1}=\exp (-i \alpha \Delta z) \psi^{s}
$$

Note that the Crank-Nicolson scheme leads to a solution of (11) correct up to second order in $\alpha \Delta z$, and also that, in the absence of any absorption, the norm of the field is preserved.

From (9) it follows that the correct propagation $(\Delta n=0)$ of the field $\psi$ would be described by:

$$
\psi^{s+1}=\exp \left\{-i k_{0}\left(n_{e}-n_{0}\right) \Delta z\right\} \psi^{s} .
$$

Comparing (15) and (13) it follows that the following requirement should be fulfilled:

$$
\text { i. } n_{0} \simeq n_{e, l}
$$

where $n_{e, l}(l=1,2, \ldots)$ are the effective refractive indexes of the modes involved. Equation (16) is a fundamental requirement and limits the general applicability of the algorithm. Possible errors introduced by deviations of (16) follow from comparison of (13) and (15), and the following should hold:

$$
|\alpha \Delta z| \ll 1 \text {, or } k_{0} \Delta z \ll 1 /\left|n_{e}-n_{0}\right|
$$

Indeed, if a single mode is launched into a $z$-invariant structure and $n_{0}=n_{e}$ the step size, $\Delta z$, may be extremely large.

From (16) it may be deduced that the propagation should be rather close to paraxial. We consider the propagation of a single mode in a waveguide making an angle of a few degrees with the $z$ axis. The modal field can be decomposed into modes belonging to the corresponding $z$-invariant structure for each step $\Delta z$. Using the FDBPM it follows that the simulated propagation is correct only if (16) holds for the latter modes.

Another requirement for FDBPM is as follows.

ii. the eigenvalue equation (8) associated with the wave equation (1) should give sufficiently accurate results, both with respect to the mode index, $n_{e}$, and the modal field. For this it is of importance to choose proper interface conditions. 


\section{Interface Conditions}

In a uniform medium the replacement of the operator $\partial^{2} / \partial x^{2}$ by a three-point difference operator is correct up to second order in $\Delta x$. Now we consider an interface coinciding with a mesh point, say at $x_{j}=j . \Delta x$. Discretization of the modal field equation (8), also using (2) leads to:

$$
\begin{aligned}
\xi_{j+1}+\xi_{j-1} & -2 \xi_{j}-d_{j} \xi_{j}+d_{j} \xi_{j-1} \\
& +\left(k_{0} \Delta x\right)^{2}\left(n_{j, a}^{2}-n_{e}^{2}\right) \xi_{j}=0 .
\end{aligned}
$$

Here $d_{j}$ should be chosen such that (18) is correct up to first order in $\Delta x$ and $n_{j, a}$ is the refractive index at the interface which should be adjusted such that (18) holds up to second order in $\Delta x$. The fields near the interface should, according to (1), behave like $\exp \left\{ \pm \gamma_{p}\left(x-x_{j}\right)\right\}, p=r, l$ for the right and left side of the interface, respectively, with $\gamma_{p}^{2}=k_{0}^{2}\left(n_{e}^{2}-n_{p}^{2}\right)$. Substituting the fields at $x_{j-1}$ and $x_{j+1}$ into (18) (in terms of $\xi_{j}$ ), expanding the exponentials up to the third term and collecting terms proportional to $\Delta x^{2}$ we arrive at:

$$
\left\{\left(d_{j}+1\right) \gamma_{l}^{2} \Delta x^{2} / 2+\gamma_{r}^{2} \Delta x^{2}+\left(k_{0} \Delta x\right)^{2}\left(n_{j, a}^{2}-n_{e}^{2}\right)\right\} \xi_{j}=0
$$

The terms up to first order in $\Delta x$ lead to:

$$
\left(\frac{\partial \xi}{\partial x}\right)_{r}-\left(\frac{\partial \xi}{\partial x}\right)_{l}\left(d_{j}+1\right)=0
$$

with

$$
\begin{aligned}
& d_{j}=0(\mathrm{TE}) \text { and } \\
& d_{j}=\left(n_{r}^{2}-n_{l}^{2}\right) / n_{l}^{2}(\mathrm{TM}) .
\end{aligned}
$$

The choice for $d_{j}$ leads to the correct interface conditions: $\xi_{l}=$ $\xi_{r}$ (TE,TM), $(\partial \xi / \partial x)_{l}=(\partial \xi / \partial x)_{r}$ (TE) and $n_{r}^{2}(\partial \xi / \partial x)_{l}=$ $n_{l}^{2}(\partial \xi / \partial x)_{r}(\mathrm{TM})$.

Equation (19) leads to

$$
n_{j, a}^{2}=\left(n_{r}^{2}+n_{l}^{2}\right) / 2+d_{j}\left(n_{l}^{2}-n_{e}^{2}\right) / 2 .
$$

Application of (21)-(22) gives rapid convergence of $n_{e}$ as a function of $\Delta x$ if used for the solution of the eigenvalue problem in a FD way.

The general case that the interface lies between two mesh points can be treated similarly. Suppose there is an interface between $x_{j}$ and $x_{j+1}$ at a distance $\zeta . \Delta x$ from $x_{j}$. In the same spirit as above it can be found:

$$
\begin{gathered}
n_{j, a}^{2}=\left(n_{j}^{2}+n_{j+1}^{2}\right) / 2+d_{j}\left(n_{j}^{2}-n_{e}^{2}\right) / 2+\zeta\left(n_{j}^{2}-n_{j+1}^{2}\right) \\
+\zeta d_{j}\left(n_{j}^{2}-n_{e}^{2}\right)+\zeta^{2}\left(n_{j+1}^{2}-n_{j}^{2}\right) / 2
\end{gathered}
$$

with

$$
\begin{aligned}
& d_{j}=0(\mathrm{TE}) \text { and } \\
& d_{j}=(1-\zeta)\left(n_{j+1}^{2}-n_{j}^{2}\right) / n_{j}^{2}(\mathrm{TM})
\end{aligned}
$$

and

$$
\begin{aligned}
n_{j+1, a}^{2}= & n_{j+1}^{2}+d_{j+1}\left(n_{e}^{2}-n_{j+1}^{2}\right) / 2+\zeta d_{j+1}\left(n_{j+1}^{2}-n_{e}^{2}\right) \\
& +\zeta^{2}\left(n_{j}^{2}-n_{j+1}^{2}\right) / 2+d_{j+1} \zeta^{2}\left(n_{j}^{2}-n_{j+1}^{2}\right) / 2(25)
\end{aligned}
$$

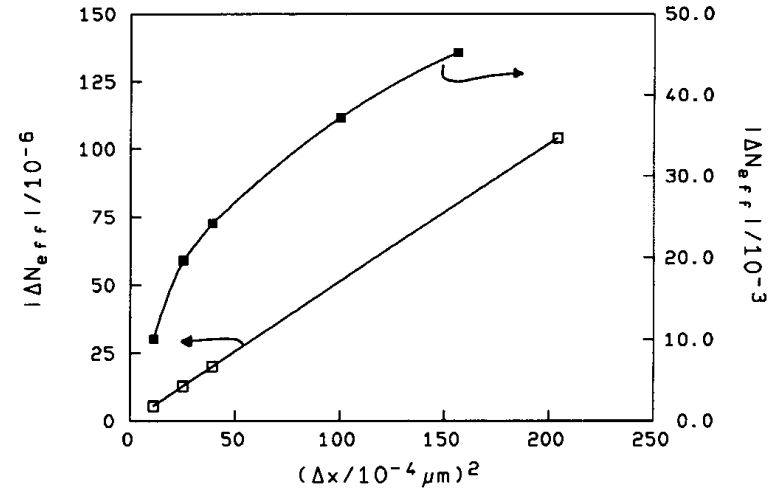

Fig. 1. Deviations of the mode index (TM), calculated using (21) and (22) (open squares) and the mean of the squared refractive indexes (black squares), from the exact value as a function of the squared lateral stepsize, $\Delta x^{2}$. The 2-D structure consists of a single interface and the refractive indexes are $n_{l}=i .4 .24$ and $n_{r}=1.5$, and the wavelength $\lambda=0.6328 \mu \mathrm{m}$

with

$$
\begin{aligned}
d_{j+1} & =0(\mathrm{TE}) \text { and } \\
d_{j+1} & =\zeta\left(n_{j+1}^{2}-n_{j}^{2}\right) /\left(n_{j+1}^{2}+\zeta n_{j}^{2}-\zeta n_{j+1}^{2}\right) \\
& =n_{j+1}^{2} /\left\{n_{j}^{2}\left(d_{j}+1\right)\right\}-1(\mathrm{TM}) .
\end{aligned}
$$

Note that application of (23) or (25) for TM modes requires (approximate) knowledge of the magnitude of the mode index. This holds also for the special case $(\zeta=0)$ described by (20)-(22). We remark that formulae (23)-(26) cannot be used in the presence of a second interface in the interval $\left(x_{j-1}, x_{j+1}\right)$ without detailed information on the modes involved. So, in order to use (23)-(26) the stepsize $\Delta x$ should be chosen small enough so that more than one mesh point lies in a given layer.

Many authors place the interface half way between adjacent grid points. (e.g., see [12]). Inspecting (23) it follows that still some accuracy can be gained by applying (23) (as $n_{j, a}^{2} \neq n_{j}^{2}$ ) for this case $(\zeta=1 / 2)$.

\section{RESULTS}

We consider first a so-called surface polariton (TM) in the presence of a single interface (see caption Fig. 1). In our calculations the interface coincides with a mesh point. Using (21) and (22) it follows from Fig. 1 that the eigenvalue converges rapidly and proportional to $\Delta x^{2}$, as might be expected. If the index at the interface $n_{j}^{2}=\left(n_{j-1}^{2}+n_{j+1}^{2} / 2\right.$ the convergence is much slower and the error in the effective refractive index contains also terms proportional in $\Delta x$ which dominate here due to the large contrast. The use of the equations of II-C in FDBPM gives quite good results for a structure containing silver, e.g., surface polaritons are propagated with the correct phase and without significant change in the modal field profile. It is of importance to have a good guess of the effective index and one should, also for reasons mentioned above, chose $n_{0} \simeq n_{e}$ in FDBPM.

In a second example we show that by the use of the proper interface conditions (23)-(26) the mode index depends only 


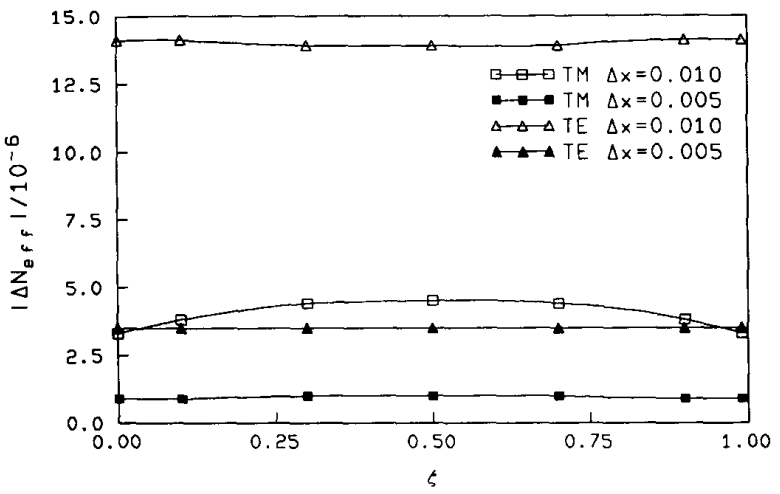

Fig. 2. Deviations of the fundamental mode index from the exact value as a function of the position of the interfaces, calculated according to (22). Units for the stepsize $\Delta x$ are $\mu \mathrm{m}$. The structure consists of a single layer, with a thickness of $1 \mu \mathrm{m}$ and $n=2$, the refractive index of the adjacent layers is $n=1$ and $\lambda=0.6328 \mu \mathrm{m}$

slightly on the position of the interface (see Fig. 2). This is of importance for the FDBPM where normally, to prevent staircase-like structures due to the discretization, smoothing, and so a certain inaccuracy, has to be introduced in the presence of structures having interfaces not parallel to the $z$ axis. Omission of (23)-(26), without any smoothing, would lead, for the structure described in the caption of Fig. 2, to relatively large errors in the refractive index of about 0.0004 if the stepsize $\Delta x=0.01 \mu \mathrm{m}$.

\section{CONCLUDING REMARKS}

We have discussed the applicability of the FDBPM and the effect of introducing adjusted refractive indexes in the vicinity of interfaces. The latter can be performed such that the discretisized wave equations are correct up to second order in the lateral stepsize $\Delta x$. It has been shown that the interface conditions lead to a much higher accuracy (for a given $\Delta x$ ) with respect to the effective mode index and so to the corresponding modal fields. The theory outlined here holds in 2D. Unfortunately, if a third coordinate is introduced the formalism cannot be used without making further assumptions on how to distribute a given curvature among the two lateral directions.

\section{REFERENCES}

[1] J. van Roey, J. van der Donk, and P. E. Lagasse, "Beam-propagation method: Analysis and assessment," J. Opt. Soc. Amer., vol. 71, no. 7, pp. $423-429,1981$

[2] L. Thylen, "The beam propagation method: An analysis of its applicability," Opt. Quantum Electron, vol. 15, pp. 433-439, 1983.

[3] P. E. Lagasse and R. Baets, "Application of the propagating beam methods to electromagnetic and acoustic wave propagation problems: A review," Radio Science, vol. 22, no. 7, pp. 1225-1233, 1987.

[4] D. Yevick and B. Hermansson, "Efficient beam propagation techniques," J. Quantum Electron., vol. 26, pp. 109-112, 1990.

[5] Y. Chung and N. Dagli, "An assessment of finite difference beam propagation method," IEEE J. of Quantum Electron., vol. 26, no. 8, pp. $1335-1339,1990$

[6] R. Accornero et al., "Finite difference methods for the analysis of integrated optical waveguides," Electron. Lett., vol. 26, no. 23, pp. $1959-1960,1990$.

[7] A. W. Snyder and J. D. Love, Optical Waveguide Theory. London Chapman and Hall, 1983.

[8] G. Dahlquist and A. Björk, Numerical Methods. Englewood Cliffs, NJ: Prentice-Hall, 1974.

[9] G. R. Hadley, "Transparent boundary condition for beam propagation," Opt. Lett., vol. 16, no. 9, pp. $624-626,1991$.

[10] R. Clauberg and P. von Allmen, "Vectorial beam propagation method for integrated optics," Electron. Lett., vol. 27, no. 8, pp. 654-655, 1991.

[11] H. Diestel, "A method for calculating the guided modes of strip-loaded optical waveguides with arbitrary index profile," IEEE J. Quantum Electron., vol. 20, no. 11, pp. $1288-1293,1984$

[12] M. S. Stern, "Semivectorial polarized finite difference method for optical waveguides with arbitrary index profiles," IEE Proc., vol. 135, Pt. $J$ no. 1 , pp. $56-63,1988$

H. J. W, M. Hoekstra, photograph and biography not available at the time of publication.

G. J. M. Krijnen, photograph and biography not available at the time of publication.

P. V. Lambeck, photograph and biography not available at the time of publication. 